Ciencia y Salud, Vol. III, No. 1, enero-abril, 2019 • ISSN: 2613-8816 | E-ISSN: 2613-8824

DOI: https://doi.org/10.22206/cysa.2019.v3i1.pp15-21

\title{
BROTE DE INFECCIÓN RESPIRATORIA AGUDA GRAVE, SANTO DOMINGO NORTE, REPÚBLICA DOMINICANA, AGOSTO 2016
}

\author{
Outbreak of severe acute respiratory infection, \\ Santo Domingo Norte, Dominican Republic, August 2016
}

\section{Manuel Colomé-Hidalgo*; Manuel Gil Fernández ${ }^{* *}$; Claribel Silfa***}

Fecha de recibido: junio 6, 2018 • Fecha de aprobado : octubre 4, 2018

Cómo citar: Colomé-Hidalgo M, Gil Fernández M, Silfa C. Brote de infección respiratoria aguda grave, Santo Domingo Norte, República Dominicana, agosto 2016. cysa [Internet]. 10 abr. 2019 [citado 12abr.2019];1(1):15-1. Disponible en: https://revistas.intec.edu.do/index.php/cisa/article/view/1326

\section{Resumen}

Antecedentes: Se realizó la investigación con el objetivo de determinar el alcance de un brote de infección respiratoria aguda grave, ocurrido en el municipio de Santo Domingo Norte en República Dominicana. Los objetivos de la investigación fueron examinar las características epidemiológicas y descriptivas de los casos, generar hipótesis (posible fuente de infección), tomar muestras para diagnóstico por laboratorio e implementar medidas de control.

Métodos: estudio descriptivo de un brote epidémico por infección respiratoria aguda grave. Se evaluaron variables sociodemográficas, signos y síntomas, datos de laboratorio y evolución clínica de los afectados. Se realizó búsqueda activa y se adoptaron medidas de contención.

Resultados: se detectaron 22 casos, teniendo al hacinamiento como principal factor de riesgo. No hubo

\footnotetext{
* Gerente del Servicio de Epidemiología, Hospital Pediátrico Dr. Hugo Mendoza, Profesor Área de la Salud INTEC, República Dominicana. Programa de Doctorado en Epidemiología y Salud Pública, Universidad Rey Juan Carlos.

Email: manuel.colome@hhm.gob.do
}

defunciones. La población más afectada correspondió a menores de un año de edad con un $50 \%$. La afectación fue igual en ambos sexos.

Conclusión: el aislamiento de virus sincitial respiratorio, parainfluenza y adenovirus tipo 3 en muestras respiratorias confirmó la presencia de un brote. Destacamos la necesidad de intensificar la vigilancia y aplicar recomendaciones de vacunación en personas con factores de riesgo, especialmente en la población infantil.

Palabras clave: Brote de enfermedades; enfermedades del tracto respiratorio; pediatría, epidemiología; salud pública

\section{Abstract}

Background: The investigation was conducted with the objective of determining the scope of an outbreak of severe acute respiratory infection, occurred in the municipality of Santo Domingo Norte in the Dominican Republic.

\footnotetext{
** Médico Salubrista, Dirección General de Epidemiología (DIGEPI). E-mail: Manuel.gil@ministeriodesalud.gob.do

*** Pediatra Infectóloga, Gerente del Departamento de Pediatría, Hospital Pediátrico Dr. Hugo Mendoza.

E-mail: Claribel.silfa@hhm.gob.do
} 
The objectives of the research were to examine the epidemiological and descriptive characteristics of the cases, generate hypotheses (possible source of infection), take samples for laboratory diagnosis and implement control measures.

Methods: descriptive study of an epidemic outbreak due to severe acute respiratory infection. Sociodemographic variables, signs and symptoms, laboratory data and clinical evolution of those affected were evaluated. Active search was carried out and containment measures were adopted.

Results: 22 cases were detected, with overcrowding as the main risk factor. There were no deaths. The most affected population corresponded to children under one year of age with $50 \%$. The affectation was the same in both sexes.

Conclusion: the isolation of respiratory syncytial virus, parainfluenza and adenovirus type 3 in respiratory samples confirmed the presence of an outbreak. We emphasize the need to intensify surveillance and apply vaccination recommendations for people with risk factors, especially in children.

Keywords: Disease outbreaks; respiratory tract infections; pediatrics; epidemiology; public health

\section{Introducción}

Las infecciones respiratorias representan un riesgo substancial para la humanidad debido a su elevado potencial de transmisión, produciendo alta morbilidad y, para formas graves, presentan elevadas tasas de hospitalización y letalidad. Las infecciones superiores son muy frecuentes pero rara vez ponen en peligro la vida mientras las infecciones respiratorias bajas son responsables de cuadros más graves de influenza, neumonía y bronquiolitis que contribuyen de forma importante a la mortalidad ${ }^{1}$.

La infección respiratoria aguda grave (IRAG) puede tener origen viral y/o bacteriano y su manejo requiere tratamiento intrahospitalario. Entre los principales agentes etiológicos que pueden causar IRAG se encuentran el Virus Sincitial Respiratorio (VSR), Parainfluenza, Influenza, Adenovirus, Streptococcus pneumoniae, Haemophilus influenzae, Mycoplasma spp, Chlamydia spp y Staphylococcus spp, entre otros. Las bacterias juegan un papel importante cuando existen factores de riesgo asociados como desnutrición, bajo peso al nacer y parto prematuro, colonización temprana de gérmenes en la orofaringe, el hacinamiento, elementos climáticos, entre otros ${ }^{2,3}$.

La transmisión puede ocurrir por gotitas de secreciones respiratorias, contacto directo de persona a persona o fómites contaminados. El periodo de transmisibilidad persiste mientras está presente el agente, iniciando en algunas ocasiones 24 horas antes de las manifestaciones clínicas, y finalizando 24-48 horas después del tratamiento y en algunos casos hasta por más de cinco días. El periodo de incubación varía dependiendo del agente etiológico, teniendo un promedio de 1-10 días. El cuadro clínico incluye: fiebre mayor de $38^{\circ} \mathrm{C}$, dificultad respiratoria, tos, hipoxia, compromiso sistémico (letargia, convulsiones, falla ventilatoria); dependiendo de la severidad del cuadro. La población con mayor riesgo de mortalidad son personas en edades extremos de la vida (niños y ancianos), embarazadas e inmunocomprometidos ${ }^{4,5,6,7}$.

El 23 de agosto del 2016, el Servicio de Epidemiología del Hospital Pediátrico Dr. Hugo Mendoza, notificó a la Dirección General de Epidemiología (DIGEPI) y al Departamento de Epidemiologia de la Dirección Municipal del Área III de Salud (DAS-III) el incremento del número de hospitalizaciones por neumonía adquirida en la comunidad (siete casos) relativo al mismo periodo en el año anterior. El día 26 de agosto, fueron hospitalizados ocho casos adicionales, también pertenecientes a Santo Domingo Norte. Los casos fueron detectados por el servicio de epidemiología hospitalaria y ante eso, se decide notificar a la DAS-III e iniciar una investigación.

\section{Objetivos}

- Determinar la magnitud del brote en tiempo, lugar y persona. 
- Describir las manifestaciones clínicas.

- Identificar el agente etiológico y la fuente de infección.

- Adoptar medidas de prevención y control a corto, mediano y largo plazo.

\section{Materiales y métodos}

Se realizó un estudio observacional descriptivo para caracterizar en tiempo, lugar y persona el brote de IRAG en Santo Domingo Norte (SDN), ocurrido entre la Semana Epidemiológica (SE) 32 y 34 del año 2016. La información se obtuvo de las historias clínicas, de los registros estadísticos y del laboratorio del hospital, además del Laboratorio Nacional de Salud Pública Dr. Defilló del Ministerio de Salud Pública.

\section{Definiciones de caso}

Se realizó una búsqueda activa en la comunidad utilizando las siguientes definiciones de caso: Caso sospechoso: Fiebre o historial de fiebre $\geq 38^{\circ} \mathrm{C}$, tos, inicio en los últimos 10 días de la fecha de atención y que requiera de hospitalización. Caso confirmado: Todo caso sospechoso con una prueba de laboratorio positiva para IRAG.

\section{Pruebas de laboratorio}

Se tomaron muestras de sangre para biometría hemática y radiografía de tórax. Se realizó hisopado nasal y orofaríngeo de las secreciones de vías respiratorias a los casos que cumplieron con los criterios de vigilancia. Las muestras obtenidas para detectar la presencia de antígenos víricos se procesaron mediante la técnica de inmuno-fluorescencia indirecta.

\section{Trabajo de campo}

El Departamento de Epidemiología de la DASIII realizó una búsqueda activa comunitaria en la zona de residencia de los afectados que cumplieron con la definición de caso de IRAG. Se recogieron variables clínicas, sociodemográficas, higiénico-sanitarias y antecedentes recientes de viajes o desplazamientos. La búsqueda activa se efectuó en las áreas del municipio donde se identificaron casos de IRAG según reportes hospitalarios. Todo caso que cumpliera con cualquier definición de caso sería referido a un centro de salud para su valoración médica y seguimiento.

\section{Análisis}

Se elaboró una base de datos utilizando la hoja de cálculo Microsoft Excel 2016 y se realizó un análisis estadístico descriptivo basado en medidas de tendencia central (media, mediana) y de dispersión (rango y desviación estándar) mediante el software Epi-Info 7.

\section{Consideraciones éticas}

En el presente estudio no se manejaron datos individuales y en las bases de datos analizadas las variables de identificación personal fueron omitidas; de igual manera, no se publicaron datos de personas, garantizando la confidencialidad. Se solicitó el consentimiento a los participantes antes de tomar muestras biológicas, las muestras fueron para uso exclusivo de este estudio y los resultados de las pruebas solo se compartieron con los involucrados.

\section{Resultados}

\subsection{Distribución en el tiempo}

Los casos comenzaron a ser captados en el centro en la semana 32. Anteriormente no se contaba con casos de IRAG notificados por la DAS-III; de igual forma, se observan dos picos intermitentes entre la SE 33 y 34, alcanzando un máximo en la semana 34, semana en la cual se obtiene la confirmación del brote. Los últimos casos se presentaron en la semana 35. (Gráfico 1) 
Gráfico 1. Curva epidémica por día de inicio de síntomas, SDN, SE 32-34 de 2016

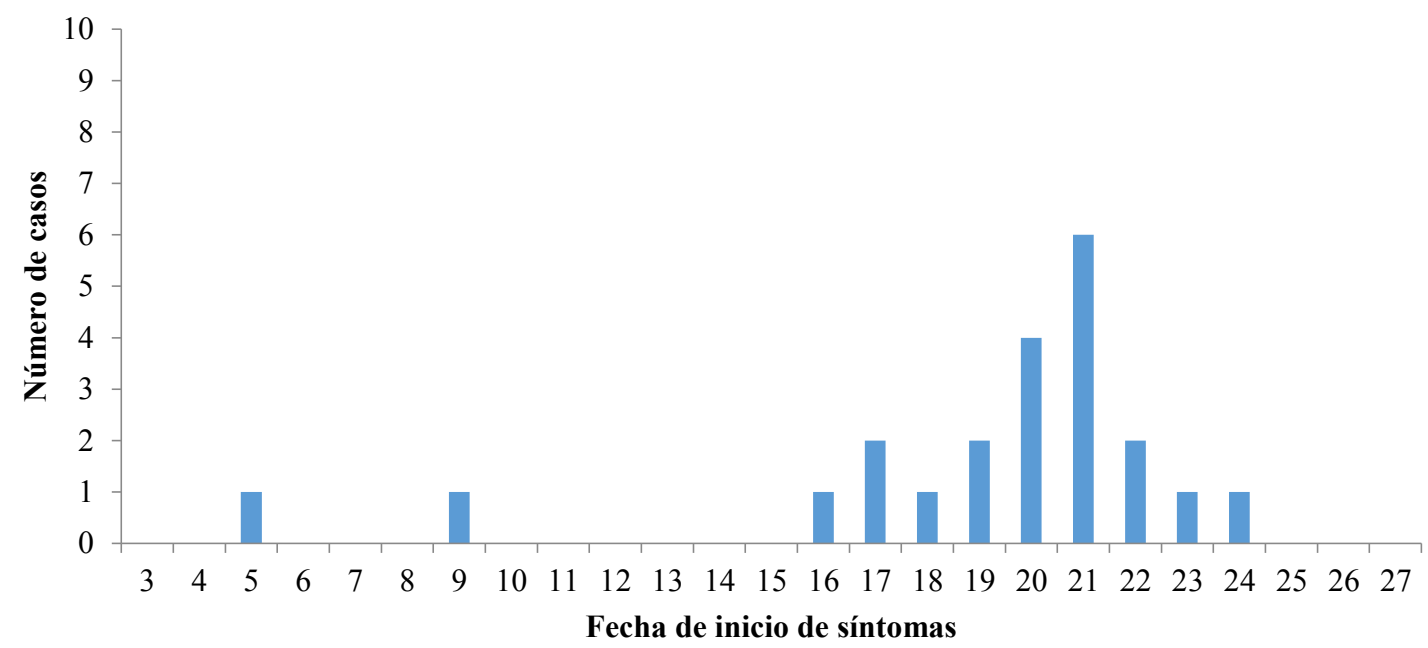

Fuente: Elaboración propia

\subsection{Lugar}

Los casos procedían de zonas ubicadas en el casco urbano de Santo Domingo Norte distribuidos en Sábana Perdida 77\% (17) y Guaricano 14\% (3), a excepción de dos que pertenecían a la parte limítrofe del municipio, correspondiente al Higüero $9 \%$. Ninguno de ellos guardaba relación entre sí de acuerdo con las investigaciones de campo efectuadas por la DAS-III. Se encuestaron individuos, distribuidos según la localización de los casos de IRAG que cumplieron con la definición de caso. La búsqueda activa comunitaria arrojó un total de tres casos que cumplían con la definición de caso planteada.

\subsection{Persona}

El $50 \%$ de los casos son del sexo femenino. La media de edad de los casos fue de 1,8 ańos, con una desviación estándar de \pm 3.4 ( (rango $=0$ a 14 años). La sintomatología más frecuente fue fiebre (95\%), tos $(95 \%)$ y dificultad respiratoria (73\%). (Cuadro 2)
Cuadro 2. Distribución de los signos y síntomas, Santo Domingo Norte, SE 32-34 de 2016

\begin{tabular}{lcc}
\hline Signos y síntomas & Frecuencia & Porcentaje \\
\hline Fiebre $>38^{\circ} \mathrm{C}$ & 21 & $95 \%$ \\
Tos & 21 & $95 \%$ \\
Dificultad respiratoria & 16 & $73 \%$ \\
Malestar general & 7 & $32 \%$ \\
Náuseas y vómitos & 5 & $22 \%$ \\
Pérdida de apetito & 3 & $14 \%$ \\
\hline
\end{tabular}

Fuente: Elaboración propia

Gráfico 2. Distribución de frecuencia por edad y sexo, SDN, SE 32-34 de 2016

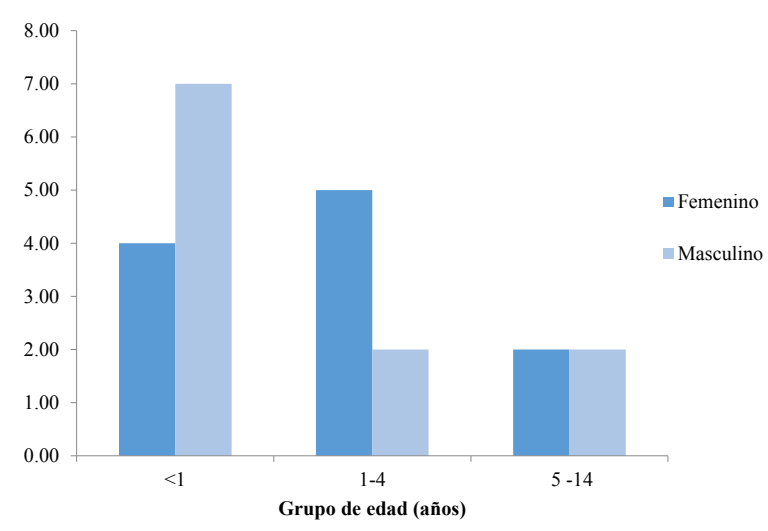

Fuente: Elaboración propia 
Cuadro 3. Distribución de la tasa de ataque según edad, SDN, SE 32-34 de 2016

\begin{tabular}{lccccc}
\hline Grupo de edad & \multicolumn{2}{c}{ Número de casos } & \multicolumn{2}{c}{ Tasa de ataque (\%) } & $\begin{array}{c}\text { Razón de ataque Mascu- } \\
\text { lino/ Femenino }\end{array}$ \\
\cline { 2 - 5 } & Femenino & Masculino & Femenino & Masculino & 1.6 \\
\hline$<1$ año & 4 & 7 & 0.07 & 0.12 & 4.2 \\
1-4 años & 5 & 2 & 0.02 & 0.10 & 1.0 \\
5-14 años & 2 & 2 & 0.00 & 0.00 & 6.8 \\
Total & 11 & 11 & 0.09 & 0.22 & \\
\hline
\end{tabular}

Fuente: Elaboración propia

\section{Laboratorio}

De un total de 7 muestras enviadas al laboratorio nacional, cinco resultaron positivas para IRAG, en tres de ellas fue aislado el Virus Sincitial Respiratorio y se obtuvo una muestra positiva respectivamente para otros virus como parainfluenza y adenovirus tipo 3, para un $71.4 \%$ de positividad.

Medidas de prevención y control aplicadas de forma inmediata

La DAS-III dirigió acciones a nivel comunitario para fortalecer las medidas generales de prevención, protección personal, identificación oportuna de signos y síntomas de IRAG, información sobre la situación de la enfermedad en la comunidad. A nivel hospitalario, el Servicio de Epidemiología aplicó un aislamiento por cohorte a los casos, se instruyó al personal de salud adoptar precauciones estándar y de transmisión por gotitas para prevenir brote nosocomial. Los pacientes estuvieron en seguimiento hasta el alta. Las medidas preventivas intrahospitalarias incluyeron una capacitación al personal de salud sobre el protocolo de vigilancia de IRAG que incluyó: definiciones de caso, notificación, recolección y transporte de muestras, tratamiento, así como también capacitación a los familiares sobre el lavado de manos ${ }^{8}$.

\section{Discusión}

La IRAG mostró una actividad viral inusual en esta época del año, al revisar los datos del comportamiento de la IRA en el territorio nacional y el comportamiento internacional. En República Dominicana durante el año 2016 se reportaron 2,762,802 episodios de enfermedad febril respiratoria aguda, correspondiente 516,248 con afecciones de las vías bajas. El número de casos de IRAG se mantiene entre lo esperado y de acuerdo con la circulación de IRAG se esperaba que se presentaran brotes aislados o conglomerados. A través de la notificación rutinaria semanal a la DIGEPI se reportaron 34,437 episodios de enfermedad febril respiratoria aguda, de los cuales 7,370 corresponden a afecciones de las vías respiratorias bajas, evidenciando la circulación de otros virus respiratorios comunes: VSR, Parainfluenza y Adenovirus. Desde la semana 46 no se ha identificado la circulación del virus de Influenza A. ${ }^{9,10}$

El brote presentado se asocia con el aumento de la circulación del virus de la influenza, VSR y parinfluenza en el país. Los casos notificados inicialmente por el hospital en la comunidad de la Javilla de Sabana Perdida correspondían a la definición planteada en el protocolo de vigilancia de IRAG. No se presentó ninguna defunción sospechosa de IRAG durante el periodo de investigación. No se evidencia en lo evaluado la presencia de conglomerados de casos en otras comunidades de SDN, aunque se ha observado nuevos casos sospechosos de IRAG este año. Los casos hospitalizados evolucionaron favorablemente y fueron tratados con el tratamiento correspondiente. 


\section{Limitaciones}

Durante el brote no se pudo obtener prueba de reacción en cadena de polimerasa en tiempo real, para confirmación, caracterización y sub-tipificación debido a que el Laboratorio Nacional no contaba con el reporte de los resultados en la plataforma del Sistema Nacional de Vigilancia Epidemiológica (SINAVE), luego esta situación fue corregida. No se pudo realizar la georreferenciación de los casos "in situ" ya que el equipo que realizó el trabajo de campo no contaba en el momento con un Sistema de Posicionamiento Global, por lo que se utilizó la plataforma de Google Maps para identificar los casos según sector de residencia en función de la dirección recogida en la ficha de investigación de brote.

\section{Conclusiones}

- Se confirmó la existencia de un brote de IRAG en el municipio de Santo Domingo Norte, afectando diferentes comunidades: Sabana Perdida, Guaricano y el Higüero. El 77\% procedentes de Sabana Perdida; de este porcentaje, los casos estaban distribuidos entre la Javilla y la Barquita.

- A la fecha de la intervención se registraron 22 casos. Entre las semanas epidemiológicas 32 y 34 se evidenció un aumento en la notificación de casos de IRAG en SDN; paralelamente en el país también se presentó también un aumento en la notificación de casos por este evento.

- No hubo diferencias en cuanto a la distribución de la enfermedad, afectando igualmente ambos sexos. La mayoría de los casos correspondieron a niños menores de un año de edad.

- De acuerdo a los reportes de laboratorio los agentes etiológicos aislados fueron tres: Virus Sincitial Respiratorio, Parainfluenza y Adenovirus tipo 3.

\section{Recomendaciones}

Se recomienda implementar un plan de acción integral para mitigar la transmisión, reducir la demanda de atención por afecciones respiratorias leves, evitar las complicaciones y la prevención y control de nuevos brotes, tanto a nivel comunitario como en el centro de salud, así como también diseñar estrategias comunicación de riesgo en la comunidad que permitan además de informar a la población la situación, dar recomendaciones claves de promoción y prevención.

\section{Agradecimientos}

Se agradece a la enfermera de vigilancia epidemiológica Alexandra Rivera, por su ayuda en la toma de muestras, a los técnicos Elaine Medina y Carlos Sosa, por su colaboración en la construcción de la base de datos y su análisis. Se agradece también al Departamento de Epidemiología de la Dirección Municipal de Salud SDN por su colaboración en la búsqueda de contactos.

\section{Bibliografía}

1. Rudan, I., Tomaskovic, L., Boschi-Pinto, C., Campbell, H. 2004. Global estimate of the incidence of clinical pneumonia among children under five years of age. Bull. World Health Organ. 82:895-903.

2. Mulholland K. Global burden of acute respiratory infections in children: implications for interventions. Pediatric Pulmonol. 2003; Vol. 36: 469-74.

3. García ML, Ordobas GM, Calvo RC, González AM, Aguilar RJ, Arregui SA, et al. Infecciones virales de vías aéreas inferiores en lactantes hospitalizados: etiología, características clínicas y factores de riesgo. An Esp Pediatr. 2001; Vol. 55: 101-7. 
4. Dirección General de Epidemiología. Boletín Epidemiológico No.52-2016, Santo Domingo, República Dominicana, 2016.

5. UNICEF. 2007. State of the World's Children. New York: United Nations Children's Fund. Organización Panamericana de la Salud Centro para el Control y la Prevención de Enfermedades. Protocolo genérico para la vigilancia de influenza. Washington, D.C. Diciembre 15 de 2006. Pág:1-47.

6. Pan American Health Organization. Operational Guidelines for Intensified National SARI Surveillance. OPS, julio del 2011.

7. Ferrerira FL, Bota DP, Bross A., et al. Serial evaluation of the SOFA score to predict outcome in critically ill patients. JAMA 2001; Vol. 286:1754-8, American Medical Association.

8. López P Juan, Galán G Gloria, Lancheros D Diocel, Olarte E Narda, Valderrama M
Alberto. Caracterización de la enfermedad respiratoria baja en pacientes menores de 5 años, hospitalizados en un centro de $3^{\circ}$ nivel. rev. fac.med [Internet]. 2016 July [cited 2018 Feb 17] ; Vol. 24, No. 2: 47-57. Available from: http://dx.doi.org/10.18359/rmed.2640.

9. Guidelines for environmental infection control in health-care facilities. Recommendations of CDC and the Healthcare Infection Control Practices Advisory Committee. 2003.

10. Escobar A. M, Martínez F., Ceruti E., Díaz A., Vicente M., Farías P., Torres G. y Vernal P. Etiología de las infecciones agudas del tracto respiratorio bajo (IRAB) en lactantes hospitalizados: estudios virológicos. Rev. Chil. Pediatr. Vol. 59, No. 6, 349-353, 1988

11. Normas Nacionales de Vigilancia Epidemiológica, DIGEPI, República Dominicana, 1998. 